\title{
Multi-Loop PID Control Design by Data-Driven Loop-Shaping Method
}

\author{
Masami Saeki and Ryoyu Kishi \\ Department of Mechanical System Engineering, Hiroshima University, \\ 1-4-1 Higashi-Hiroshima, 739-8527
}

Japan

\section{Introduction}

In the analysis and synthesis of control systems, model-based design methods are standard and powerful. However, the plant property is wide-ranging, and the identification of the mathematical model requires much effort and expert knowledge. Since the purpose of control design is to find a controller that optimizes a performance index using plant responses and other preliminary knowledge, a mathematical model is not necessarily required for design though it is very useful. We consider that essential merit of this data-driven design approach lies in the fact that the controller structure is known completely, whereas it is impossible to identify the plant structure without uncertainties.

Design methods that satisfy the following conditions are considered to be more user friendly. a) Plant responses used for design can be obtained in the normal plant operation. b) Not so many plant responses are required for design. For example, a few step responses may be desirable, preferably in the closed-loop operation. c) The design method is applicable to various plants by tuning one or two design parameters. $d$ ) The parameter value of the design specification has absolute meaning for control performance. Namely, it is desirable to be plant independent.

Recently, there have been two major data-driven approaches proposed. One is the iterative feedback tuning (IFT) (Hjalmarsson et al. (1999); Lequin et al. (2003)). Since IFT requires special experiments to get the plant responses iteratively, it does not satisfy the requirements a) and b). The other is the virtual reference feedback tuning (VRFT) (Campi et al. (2002)). VRFT is based on model matching, and the controller that gives a desired closed-loop transfer function is sought. We consider that VRFT almost satisfies a), b), c). Since preliminary knowledge is necessary to give an adequate and realizable target closed-loop transfer function, the requirement $d$ ) is not satisfied. VRFT is suitable for those problems where the target closed-loop transfer function can be given or easily found from some preliminary knowledge. In the classical control and robust control, loop-shaping is recognized as a very practical and useful design specification (Skogestad \& Postlethwaite (2007)). PID controller is widely used for the industrial plants and the tuning of the PID gains is easier compared with other controllers (Åström \& Hägglund (1995)). Therefore, we have developed a data driven method for the mixed sensitivity control problem of PID control (Saeki (2004a), Saeki et al. (2006)) based on unfalsified control (Safonov \& Tsao (1997)). After this, we found a simpler problem setting for PI control in the reference (Åström et al. (1998)), where the integral gain of PI controller is 
maximized subject to the maximum sensitivity condition and this problem is treated on the frequency domain. Since this problem setting and the solutions satisfy c) and d), we have studied a data-driven method for this problem in order to develop a method that satisfies all the requirements. This problem can be considered as a loop shaping problem, which will be explained in Section 2.

The basic idea of unfalsified control is to remove the controllers from the candidate controllers if they do not satisfy the design specification for given plant responses, and to apply an unfalsified controller to the plant. We have examined application of this idea to robust control design. Since we found by simulation that the falsification condition of an $L_{2}$ gain performance index cannot efficiently falsifies the controllers by a single plant response, we proposed a method of generating many virtual responses by filtering the measured data with many bandpass filters (Saeki et al. (2006)). We have obtained a data-driven method that almost satisfies a) and b) for a single-input single-output plant (Saeki (2008)). We refer to this method as the data driven loop shaping method (DDLS).

In this paper, we will study an extension of DDLS to multi-loop PID control, and we will examine the possibility of this approach because the design of multi-loop PID control systems is much harder than that of single-input single-output plants (Johnson \& Moradi (2005)). A design problem is formulated in Section 2, the constraints on PID gains are derived in Section 3 , and a method of generating plant response data and the design procedure are explained in Section 4. A numerical example for a two-input two-output time-delay plant is shown in Section 5, and an experimental result for a two-rotor hovering system is shown in Section 6. For signals $w(t) \in R^{n}, v(t) \in R^{n}, t \in[0, \infty)$, we use the following notations. $\|w\|_{2}=$ $\sqrt{\int_{0}^{\infty} w(\tau)^{T} w(\tau) d \tau},\|w\|_{2 T}=\sqrt{\int_{0}^{T} w(\tau)^{T} w(\tau) d \tau},\langle w, v\rangle=\int_{0}^{\infty} w(\tau)^{T} v(\tau) d \tau,\langle w, v\rangle_{T}=$ $\int_{0}^{T} w(\tau)^{T} v(\tau) d \tau$. Denote the $(i, j)$-element of a matrix $A$ as $[A]_{i j}$ and the $i$ th-element of a vector $b$ as $[b]_{i}$.

\section{Problem setting}

Let us consider the feedback system described by

$$
\begin{aligned}
y & =P e \\
e & =w-u \\
u & =K y
\end{aligned}
$$

where $y, e, u, w \in R^{m}$. The plant $P$ is linear time-invariant and $m$-input and $m$-output. $K$ is a multi-loop PID controller given by

$$
K(s)=K_{P}+K_{I} \frac{1}{S}+K_{D^{s}}
$$

where $K_{P}, K_{I}, K_{D}$ are constant diagonal matrices. We will use the notation $\hat{K}=\left[K_{P}, K_{I}, K_{D}\right]$. Since we are considering a data-driven method, we assume that a few input-output responses of the plant, $e(t), y(t)$, are given in the finite interval $t \in[0, T]$, where the plant is at the steady state at $t=0$, i.e., $e(t)=0, y(t)=0, t<0$. If $e(t)=e(0) \neq 0, y(t)=y(0) \neq 0$ for $t<0$, the bias must be eliminated by $e(t)-e(0), y(t)-y(0)$. These data will be used for design.

The sensitivity and complementary sensitivity functions at the plant input are denoted by

$$
\begin{aligned}
& S_{I}=(I+K P)^{-1} \\
& T_{I}=(I+K P)^{-1} K P
\end{aligned}
$$


For this system, following properties are known.

a) The maximum sensitivity defined by

$$
M_{S}=\max _{0 \leq \omega<\infty} \sigma_{\max }\left\{S_{I}(j \omega)\right\}
$$

is a useful measure for stability margin, and the typical values of $M_{s}$ are in the range of 1.2 to 2 . This condition is represented by

$$
\sigma_{\max }\left\{S_{I}(j \omega)\right\}<\gamma_{1}, \omega \in R, \gamma_{1} \in[1.2,2]
$$

In the time domain, this is equivalent to the $L_{2}$-gain condition;

$$
\|e\|_{2}<\gamma_{1}\|w\|_{2}
$$

for all $w \in L_{2}$ and $e=S_{I} w$.

b) A robust stability condition is given by

$$
\sigma_{\max }\left\{T_{I}(j \omega)\right\}<\gamma_{2}, \omega \in R
$$

, which is equivalent to the $L_{2}$-gain condition;

$$
\|u\|_{2}<\gamma_{2}\|w\|_{2}
$$

for all $w \in L_{2}$ and $u=T_{I} w$.

c) Let $y_{i}(t)$ be the response for a step disturbance $w_{i}(t)=1$ and $w_{j}(t)=0, j \neq i$. Then the intergal of $y_{i}(t)$ satisfies

$$
\int_{0}^{\infty} y_{i}(\tau) d \tau=\frac{1}{\left[K_{I}\right]_{i i}}
$$

From this property, disturbance attenuation is attained by making $\left|\left[K_{I}\right]_{i i}\right|$ larger for $i=$ $1,2, \cdots, m$. We formulate the plant description so that $\left[K_{I}\right]_{i i}>0, i=1,2, \cdots, m$ can be a necessary condition for the closed-loop stability, and, for this system, we adopt the next performance index to measure the largeness of $K_{I}$.

$$
J=\sum_{i=1}^{m}\left[K_{I}\right]_{i i}
$$

d) When $\sigma_{\min }\left\{K_{I} P(0)\right\} \neq 0$, the next approximation is satisfied at low frequencies.

$$
S_{I}(j \omega) \approx j \omega\left(K_{I} P(0)\right)^{-1}
$$

In this paper, we will study a maximization problem of the integral gain of the PID controller under the maximum sensitivity condition and, if necessary, the robust stability condition. From the above properties a), b), and c), this problem is considered as a disturbance attenuation problem with adequate stability margin. This is also considered as a loop shaping problem. Namely, from the properties a) and d), if $\sigma_{\min }\left\{K_{I} P(0)\right\} \neq 0$, the system has a loop shape illustrated in Fig. 1. By substituting (14) into $\sigma_{\max }\left\{S_{I}(j \omega)\right\}<1, \omega<\sigma_{\min }\left\{K_{I} P(0)\right\}$. Therefore, the control bandwidth is estimated by $\sigma_{\min }\left\{K_{I} P(0)\right\}$, which can be made larger by making $K_{I}$ larger. 


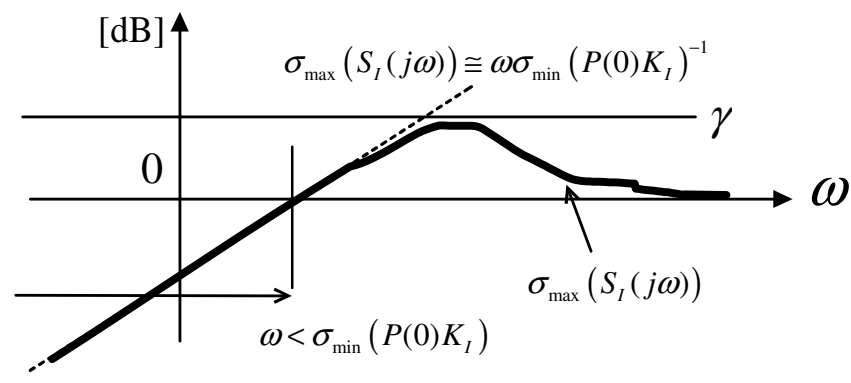

Fig. 1. Loop shaping for the sensitivity function

Lemma 1(Vidyasagar (1993)) Suppose that the system satisfies causality and it is in the steady state at $t=0$. Then, if (9) is satisfied,

$$
\|e\|_{2 T}<\gamma_{1}\|w\|_{2 T}
$$

for $T>0$. Similarly, if (11) is satisfied,

$$
\|u\|_{2 T}<\gamma_{2}\|w\|_{2 T}
$$

for $T>0$.

Compared with (9), the merit of the condition (15) is that it can be calculated for the finite length data $e(t), y(t), t \in[0, T]$, and the demerit is that (15) is only a necessary condition for (9). Since we can only use the finite length data, we will use the condition (15) instead of (9). The same idea is applied to (11) and (16). In this paper, we will examine the next design problem.

Loop-shaping problem: For the feedback system (1)-(3), find a PID controller that maximizes $J$ defined by (13) subject to

$$
\begin{gathered}
\|e\|_{2 T}<\gamma_{1}\|w\|_{2 T} \\
\|u\|_{2 T}<\gamma_{2}\|w\|_{2 T}
\end{gathered}
$$

for sufficiently many disturbances $w=w_{i} \in L_{2 e}, \quad i=1,2, \cdots, N$.

In this problem setting, it is ideal to test the constraints for all $w \in L_{2 e}$, but practically we can only generate a finite number of disturbances from the measured data $e(t), y(t)$ in the following discussion. Therefore, the number of $w$ is finite in the above problem setting.

\section{Derivation of convex constraints on PID gains}

\subsection{Derivation of a constraint from (17)}

From (17),

$$
\langle w, w\rangle_{T}>\frac{1}{\gamma_{1}^{2}}\langle e, e\rangle_{T}
$$


The disturbance $w$ that gives the plant response $e, y$ is given by

$$
\begin{aligned}
w(t) & =e(t)+u(t) \\
u(t) & =K_{P} y(t)+K_{I} y_{I}(t)+K_{D} y_{D}(t)
\end{aligned}
$$

where $y_{I}(t)=\int_{0}^{t} y(\tau) d \tau$ and $y_{D}(t)=\frac{d y}{d t}(t)$. Substitution of (20) into (19) gives

$$
\langle e+u, e+u\rangle_{T}>\frac{1}{\gamma_{1}^{2}}\langle e, e\rangle_{T}
$$

This is a concave constraint on the PID gains. Next, we will derive a linear constraint from (22) as a sufficient condition. From

$$
\left\langle u-u_{0}, u-u_{0}\right\rangle_{T} \geq 0
$$

for any $u_{0}(t)$, a sufficient condition for (22) is given by

$$
\langle e+u, e+u\rangle_{T}>\frac{1}{\gamma_{1}^{2}}\langle e, e\rangle_{T}+\left\langle u-u_{0}, u-u_{0}\right\rangle_{T}
$$

By expanding this,

$$
\left\langle e+u_{0}, u\right\rangle_{T}>b
$$

where

$$
b=\frac{1}{2}\left\{\left(\frac{1}{\gamma_{1}^{2}}-1\right)\langle e, e\rangle_{T}+\left\langle u_{0}, u_{0}\right\rangle_{T}\right\}
$$

This is a linear constraint on the PID gains. Thus, we have the next lemma by substituting (21) into (25).

Lemma 2 If the next linear constraint on the PID gains is satisfied for a data $e(t), y(t),(17)$ is also satisfied for the same data.

$$
\sum_{i=1}^{m}\left\{a_{P i}\left[K_{P}\right]_{i i}+a_{I i}\left[K_{I}\right]_{i i}+a_{D i}\left[K_{D}\right]_{i i}\right\}>b
$$

where $a_{P i}=\left\langle\left[e+u_{0}\right]_{i},[y]_{i}\right\rangle_{T}, a_{I i}=\left\langle\left[e+u_{0}\right]_{i},\left[y_{I}\right]_{i}\right\rangle_{T}$, and $a_{D i}=\left\langle\left[e+u_{0}\right]_{i},\left[y_{D}\right]_{i}\right\rangle_{T}$.

The linear constraint (27) is satisfied for any $u_{0}$, but $u_{0}$ should be chosen so that the gain set defined by the constraint may contain the set of stabilizing PID gains. We assume that a stabilizing PID gain $\hat{K}=\hat{K}_{a}$ is given. Denote $u(t)$ of $(21)$ as $u_{a}(t)$ for $\hat{K}_{a}$ and further assume that (22), which is equivalent to (17), is satisfied for $u(t)=u_{a}(t)$.

The set of $u$ that satisfies (22) corresponds to the outside region of the sphere with center $-e$ and radius $\|e\|_{2} / \gamma_{1}$ as illustrated in Fig. 2 . This set is concave and $u_{a}$ lies outside the sphere by assumption. Let $u_{0}$ be the intersection of the segment that connects $-e$ and $u_{a}$ and the sphere. We consider that the sphere is approximated by the plane which touches the sphere at the point $u_{0}$ as illustrated shown in Fig. 2 . Note that this convex set determined by the plane is described by (27) with this $u_{0}$.

Let us calculate $u_{0}$. The segment is described by

$$
u=q u_{a}+(1-q)(-e), 0 \leq q \leq 1 .
$$




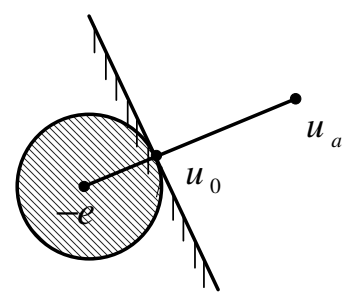

Fig. 2. Approximation of the concave region by plane

By substituting this into (22),

$$
q^{2}\left\langle e+u_{a}, e+u_{a}\right\rangle_{T}>\frac{1}{\gamma_{1}^{2}}\langle e, e\rangle_{T} .
$$

From this, the minimum value of $q$ is found to be

$$
q_{0}=\frac{1}{\gamma_{1}} \frac{\|e\|_{2 T}}{\left\|u_{a}+e\right\|_{2 T}},
$$

and

$$
u_{0}=q_{0} u_{a}-\left(1-q_{0}\right) e .
$$

From the above derivation, we have the next lemma.

Lemma 3 The stabilizing gain $K_{a}$ satisfies the linear constraint (27) for $u_{0}$ that is given by (30) and (31).

The above discussions are summarized as the next theorem.

Theorem 1 Suppose that a data $e(t), y(t), t \in[0, T]$ and a stabilizing PID gain $K_{a}$ that satisfies (17) are given. The linear constraint (27) with $u_{0}$ given by (30) and (31) is a sufficient condition for (17), and the linear constraint is satisfied for the stabilizing PID gain.

\subsection{Derivation of a constraint from (18)}

By substituting (20) into (18),

$$
\frac{1}{\gamma_{2}^{2}}\langle u, u\rangle_{T}>\langle e+u, e+u\rangle_{T}
$$

By expanding this,

$$
\left(1-\frac{1}{\gamma_{2}^{2}}\right)\langle u, u\rangle_{T}+2\langle e, u\rangle_{T}+\langle e, e\rangle_{T}>0
$$

We will derive convex constraints from (33), where three cases are considered depending on the value of $\gamma_{2}$.

If $\gamma_{2}=1$, (33) becomes

$$
2\langle e, u\rangle_{T}+\langle e, e\rangle_{T}>0
$$


From this inequality, a linear constrains on PID gains can be derived immediately. Namely,

$$
2 \sum_{i=1}^{m} P_{i} x_{i}+c>0
$$

where

$$
\begin{aligned}
& x_{i}=\left[\begin{array}{lll}
{\left[K_{P}\right]_{i i}} & {\left[K_{I}\right]_{i i}} & {\left[K_{D}\right]_{i i}}
\end{array}\right]^{T}, \\
& P_{i}=\left[\left\langle[e]_{i},[y]_{i}\right\rangle_{T},\left\langle[e]_{i},\left[y_{I}\right]_{i}\right\rangle_{T},\left\langle[e]_{i},\left[y_{D}\right]_{i}\right\rangle_{T}\right], \\
& c=\langle e, e\rangle_{T}
\end{aligned}
$$

If $\gamma_{2}>1,1-1 / \gamma_{2}^{2}>0$ and (33) can be represented as

$$
\langle u, u\rangle_{T}+\left(\frac{2 \gamma_{2}^{2}}{\gamma_{2}^{2}-1}\right)\langle e, u\rangle_{T}+\left(\frac{\gamma_{2}^{2}}{\gamma_{2}^{2}-1}\right)\langle e, e\rangle_{T}>0
$$

Further, by denoting $\tilde{e}=\left(\gamma_{2}^{2} /\left(\gamma_{2}^{2}-1\right)\right) e$, this inequality can be represented as

$$
\langle u, u\rangle_{T}+2\langle\tilde{e}, u\rangle_{T}+\langle\tilde{e}, \tilde{e}\rangle_{T}>\frac{1}{\gamma_{2}^{2}}\langle\tilde{e}, \tilde{e}\rangle_{T}
$$

Since this condition has the same form as (22), Theorem 1 with $e$ replaced with $\tilde{e}$ is satisfied. If $\gamma_{2}<1$, (33) is a convex constraint and represented as

$$
\left(1-\frac{1}{\gamma_{2}^{2}}\right) \sum_{i=1}^{m} x_{i}^{T} Q_{i} x_{i}+2 \sum_{i=1}^{m} P_{i} x_{i}+c>0
$$

where $Q_{i}=Q_{i}^{T}$ and

$$
Q_{i}=\left[\begin{array}{ccc}
\left\langle[y]_{i},[y]_{i}\right\rangle_{T} & \left\langle[y]_{i},\left[y_{I}\right]_{i}\right\rangle_{T} & \left\langle[y]_{i},\left[y_{D}\right]_{i}\right\rangle_{T} \\
& \left\langle\left[y_{I}\right]_{i},\left[y_{I}\right]_{i}\right\rangle_{T} & \left\langle\left[y_{I}\right]_{i},\left[y_{D}\right]_{i}\right\rangle_{T} \\
* & & \left\langle\left[y_{D}\right]_{i},\left[y_{D}\right]_{i}\right\rangle_{T}
\end{array}\right]
$$

By representing $Q_{i}$ by the singular value decomposition form $Q_{i}=U_{i 1}^{T} \Sigma_{i} U_{i 1}$ where $\Sigma_{i}>0$ and applying Schur complement, the next LMI (linear matrix inequality) with respect to $x_{i}{ }^{\prime} \mathrm{s}$ is obtained.

$$
\sum_{i=1}^{m}\left[\begin{array}{cc}
P_{i} x_{i}+x_{i}^{T} P_{i}^{T}+\frac{1}{m} c & x_{i}^{T} U_{i 1}^{T} \\
U_{i 1} x_{i} & \frac{\gamma_{2}^{i}}{1-\gamma_{2}^{2}} \Sigma_{i}^{-1}
\end{array}\right]>0
$$

The above discussions are summarized as the following two theorems.

Theorem 2 Suppose that a data $e(t), y(t), t \in[0, T]$ is given. If $\gamma_{2}=1,(18)$ is equivalent to the linear constraint (36). If $\gamma_{2}<1,(18)$ is equivalent to the LMI constraint (39).

Theorem 3 Suppose that a data $e(t), y(t), t \in[0, T]$ and a stabilizing PID gain that satisfies (18) are given. If $\gamma_{2}>1$, the linear constraint (27) with $u_{0}$ given by (30) and (31), where $e$ is replaced with $\tilde{e}$, is a sufficient condition for (18), and the linear constraint is satisfied for the stabilizing PID gain. 


\section{Data generation and design procedure}

\subsection{Data generation by filtering}

Since the multi-loop PID controller contains many variables to be determined, many linear constraints are necessary for the determination. Since one linear constraint (27) is derived from one input-output response $e(t), y(t), t \in[0, T]$, many input output responses would be necessary.

In order to obtain the plant response $e(t)$ and $y(t)$, we may give the test input to $w(t)$ of the system (1)-(3) at the steady state, or to the reference $r(t)$ of the system described by

$$
\begin{aligned}
& y=P e \\
& e=K(r-y) .
\end{aligned}
$$

Since the plant is $m$-input and $m$-output, $m$ sets of responses $e(t)$ and $y(t)$ may be necessary at least. Therefore, we give a test input for the $j$ th input $[w]_{j}$ or $[r]_{j}$ and measure the input-output response $\{e(t), y(t)\}$, which are denoted by $e^{j}, y^{j}$. By iterating this experiment $m$ times, $m$ sets of data $e^{j}, y^{j}, j=1,2, \ldots, m$ are obtained.

Next, we will generate many fictitious data $e^{i j}(t), y^{i j}(t), i=1,2, \ldots, n_{F}, j=1,2, \ldots, m$ by

$$
\begin{aligned}
e^{i j}(t) & =F_{i}(s) e^{j}(t) \\
y^{i j}(t) & =F_{i}(s) y^{j}(t), t \in[0, T]
\end{aligned}
$$

where the filter $F_{i}(s)$ is a stable transfer function. Note that the notation $F_{i}(s) e^{j}(t)$ means that $F_{i}(s)$ filters each element of the $m$-dimensional vector $e^{j}(t)$.

From the assumptions that $P$ is linear time-invariant and that the system is in the steady state at $t=0$,

$$
y^{i j}(t)=P(s) e^{i j}(t)
$$

is satisfied. Namely, the data $e^{i j}(t), y^{i j}(t)$ can be considered as the input-output response of the plant.

Remark 1 Even if the condition that $P$ is linear time-invariant is not assumed, the above loop shaping problem can be interpreted for a nonlinear plant as a problem with the weighted $L_{2}$ gain criterion given by

$$
\left\|F_{i}(s) e\right\|_{2}<\gamma_{1}\left\|F_{i}(s) w\right\|_{2}, i=1,2, \ldots, n_{F} .
$$

Namely, if a controller is falsified by the condition (17) for the filtered responses of a nonlinear plant, we can say that the controller is falsified by the criterion (45).

Remark 2 From the previous discussions, the $L_{2}$ gain constraint (17) is evaluated for the fictitious disturbances $w(t)$ given by (20), i.e. $w(t)=e(t)+K y(t)$ for the data $e(t)=e^{i j}(t)$, $y(t)=y^{i j}(t), i=1,2, \ldots, n_{F}, j=1,2, \ldots, m$ and the number of disturbances is $N=n_{F} m$.

\subsection{Filter selection}

We use the next bandpass filters $F_{i}(s)$ for the sample frequencies $\omega_{i}, i=1,2, \cdots, n_{F}$.

$$
\begin{aligned}
& F_{i}(s)=\hat{\psi}\left(s / \omega_{i}\right) \\
& \hat{\psi}(s)=\left(\frac{s}{(s+\alpha)^{2}+1}\right)^{4}
\end{aligned}
$$


The gain plot of $\hat{\psi}(s)$ is shown in Fig. 3. Since the peak gain is taken at $\omega=\omega_{i}\left(1+\alpha^{2}\right)^{0.5}$, this filter can be used for extracting this frequency component.

Let us consider the filtering from the viewpoint of the wavelet transform (Addison (2002)). In the last decade, wavelet transform has become popular as a time-frequency analysis tool. Wavelet transform is useful to get important information regarding the frequency properties lies locally in the time-domain from the non-stationary signals $e, y$.

If we denote the impulse response of $F_{i}(s)=\hat{\psi}\left(s / \omega_{i}\right)$ as $L^{-1}\left\{F_{i}(s)\right\}=\omega_{i} \psi\left(\omega_{i} t\right)$, then the correspondence

$$
a \leftrightarrow \frac{1}{\omega_{i}}, \quad b \leftrightarrow t,-\phi(-t) \leftrightarrow \psi(t)
$$

is satisfied between the filtering;

$$
\begin{aligned}
y_{i}(t) & =F_{i}(s) y(t) \\
& =\omega_{i} \int_{0}^{t} \psi\left(\omega_{i}(t-\tau)\right) y(\tau) d \tau .
\end{aligned}
$$

and the integral wavelet transform;

$$
\left(W_{\phi} y\right)(b, a)=|a|^{-1} \int_{-\infty}^{\infty} \phi\left(\frac{\tau-b}{a}\right) y(\tau) d \tau .
$$

The impulse response $\psi(t)$ of $\hat{\psi}(s)$ with $\alpha=0.5$ is shown in Fig. 4 , and the graph of $-\phi_{d b 10}(-t)$ is shown in Fig. 5 for the Daubechies wavelet "db10" $\phi_{d b 10}(t)$. From the uncertainty principle in the wavelet analysis, there is a trade-off between the time window and the frequency window. The time-frequency window can be tuned by the parameter $\alpha . \alpha=0.5$ is the value with which $\psi(t)$ can be close to $-\phi_{d b 10}(-t)$.

By the way, since $F_{i}(s)$ has four zeros at $s=0, F_{i}(s) e(t)=0$ for $e(t)=a_{0}+a_{1} t+a_{2} t^{2}+a_{3} t^{3}$. Namely, the output becomes zero for this class of smooth inputs. For step or ramp inputs, their time-derivatives have discontinuity and so we have nonzero outputs. For the response $e(t), y(t)$ shown in Fig. 6, the responses filtered by $F_{i}(s)$ are shown in Fig. 7.

\subsection{Design procedure}

Step 1 Measure the input output responses $e^{j}(t), y^{j}(t), t \in[0, T], j=1,2, \ldots, m$ by exciting the system at the steady state. If the response has bias, eliminate it.

Step 2 Set $\omega_{i}, i=1,2, \ldots, n_{F}$ as logarithmically equally spaced $n_{F}$ points in the important frequency range for control. Generate the fictitious responses

$$
e^{i j}(t), y^{i j}(t), t \in[0, T], i=1,2, \ldots, n_{F} .
$$

from $e^{j}(t), y^{j}(t), t \in[0, T], j=1,2, \ldots, m$ by (42) and (43). Set the value of $\gamma_{1}$. Set the value of $\gamma_{2}$ if necessary.

Step 3 Give a stabilizing PID gain $\hat{K}_{a}$ that satisfies (17) and (18) for $\gamma_{1}$ and $\gamma_{2}$. Then, compute the constraints on the PID gains for the $n_{F}$ set of responses $e^{i j}(t), y^{i j}(t)$ following Theorems 1, 2, 3 .

Step 4 If (17) is only considered as the constraints, solve a linear programming problem of maximizing $J$ subject to (13) and the linear constrains on the PID gains. Otherwise, if both (17) and (18) are considered, solve an LMI problem of maximizing $J$ defined by (13) and the linear constrains on the PID gains. 


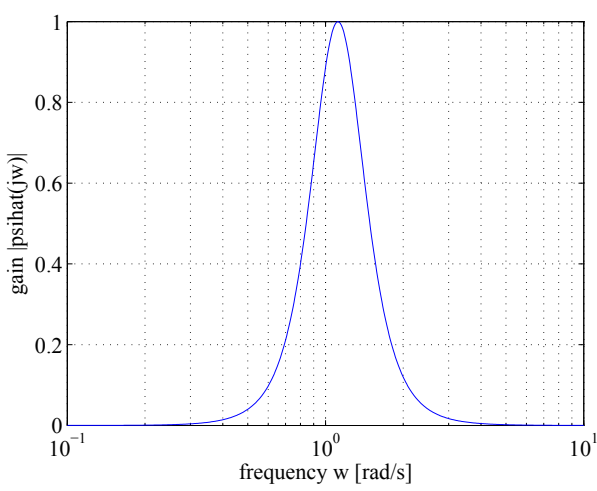

Fig. 3. Gain plot of $\hat{\psi}(j \omega)$

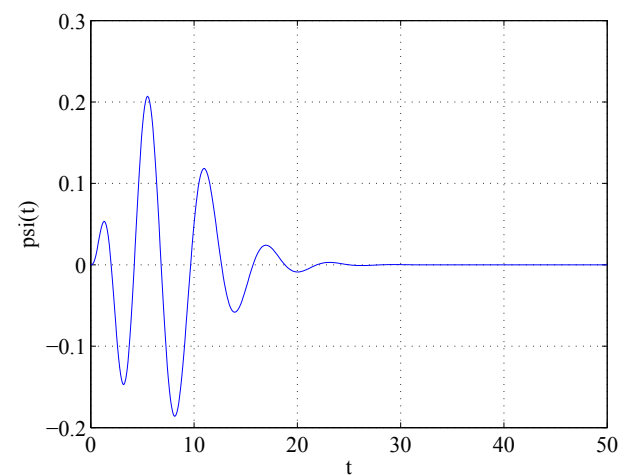

Fig. 4. Impulse response $\psi(t)$ for $\sigma=0.5$

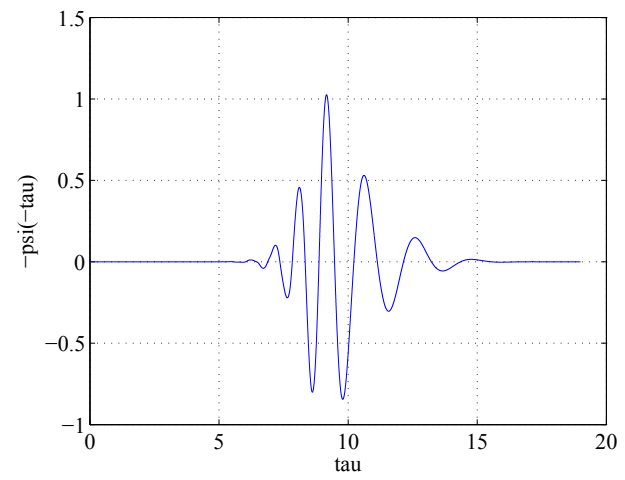

Fig. 5. Mother wavelet db10 $y=-\phi_{d b 10}(-\tau)$

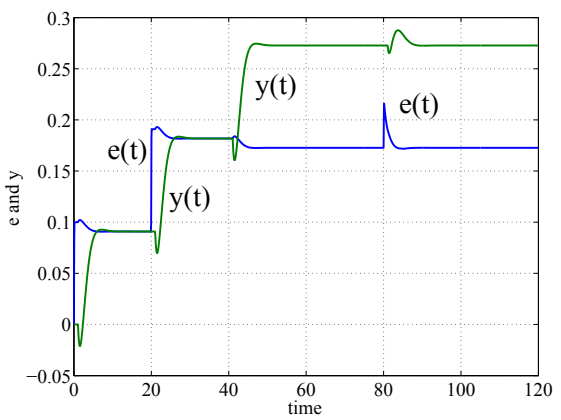

Fig. 6. $e(t)$ and $y(t)$

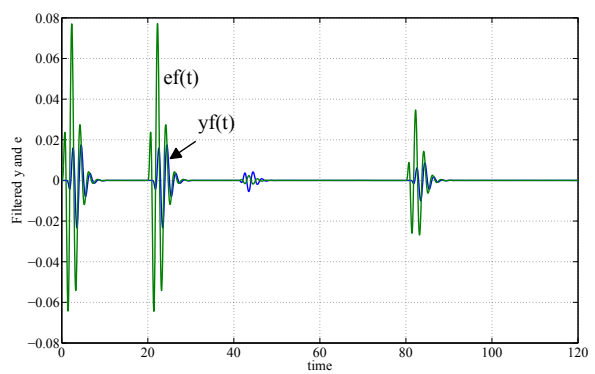

Fig. 7. $e_{f}(t)=F_{i}(s) e(t), y_{f}(t)=F_{i}(s) y(t)$ 
Step 5 Implement the PID controller.

If the plant is stable, a low gain $P$ or $P D$ controller is usually a stabilizing PID gain $\hat{K}_{a}$ that satisfies (17) and (18) in Step 3. However, if the plant is marginally stable or unstable, it may be not so easy to find such a stabilizing gain.

\section{A numerical examples for a plant with time-delay}

Let us consider the feedback system described by (40)(41), where the plant transfer function is given by

$$
P(s)=\left[\begin{array}{cc}
\frac{12.8}{1+16.7 s} e^{-s} & \frac{18.9}{1+21 s} e^{-3 s} \\
\frac{6.6}{1+10.9 s} e^{-7 s} & \frac{19.4}{1+14.4 s} e^{-3 s}
\end{array}\right] .
$$

This transfer function is obtained from that of the Wood and Berry's binary distillation column process (Wood \& Berry (1973)) by changing the sign of the $(1,2)$ and $(2,2)$ elements so that the plant may be stabilized by positive $K_{I}(1)$ and $K_{I}(2)$. Therefore, a solution for the Wood and Berry's binary distillation column process can be obtained by changing the sign of the second PI controller designed by our method.

First, we will get the plant responses with a stabilizing controller $K(s)=0.1 I_{2}$. Measurement noises with zero mean values and variances 0.0001 are given at the output $y_{1}$ and $y_{2}$ in the closed-loop operation, respectively. Fig. 8 shows the response $e(t)$ and $y(t)$ for the reference input $r_{1}(t)=1, r_{2}(t)=0$, and Fig. 9 for $r_{1}(t)=0, r_{2}(t)=1$.
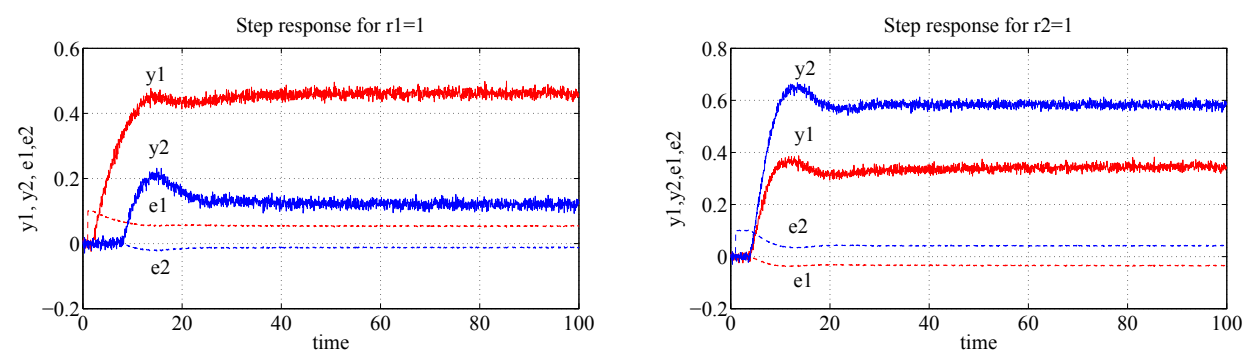

Fig. 8. Inputs and outputs of the plant for Fig. 9. Inputs and outputs of the plant for $r_{1}(t)=1$ with $K=0.1 I_{2}$ $r_{2}(t)=1$ with $K=0.1 I_{2}$

Now, design a diagonal PI controller using these step response data. We will only consider the main constraint (17), and hence a solution can be obtained by applying linear programming. We set $\gamma_{1}=1.5$ and $\omega_{i}, i=1,2, \ldots, 40$ logarithmically equally spaced frequencies between $0.1[\mathrm{rad} / \mathrm{s}]$ and $10[\mathrm{rad} / \mathrm{s}]$, and give the bandpass filters by (46). The derivative and integral calculations in the continuous time are executed approximately in the discrete time, where the sampling interval is $\Delta T=0.05[s]$. A solution that maximizes $J=\left[K_{I}\right]_{11}+\left[K_{I}\right]_{22}$ is given by

$$
K(s)=\left[\begin{array}{cc}
0.279+\frac{0.0368}{s} & 0 \\
0 & 0.0698+\frac{0.00834}{s}
\end{array}\right] .
$$

Fig. 10 shows the singular value plots of $S_{I}(s)$ and $T_{I}(s)$. In this figure, the horizontal line shows the bound $\gamma_{1}=1.5$. Note that since the condition (17) is a necessary condition for 
the $L_{2}$ gain constraint (9), the maximum singular value tends to become larger than $\gamma_{1}$. Fig. 11 shows the step response $y(t)$ for the reference input $r_{1}(t)=1, r_{2}(t)=0$, and Fig. 12 for $r_{1}(t)=0, r_{2}(t)=1$.

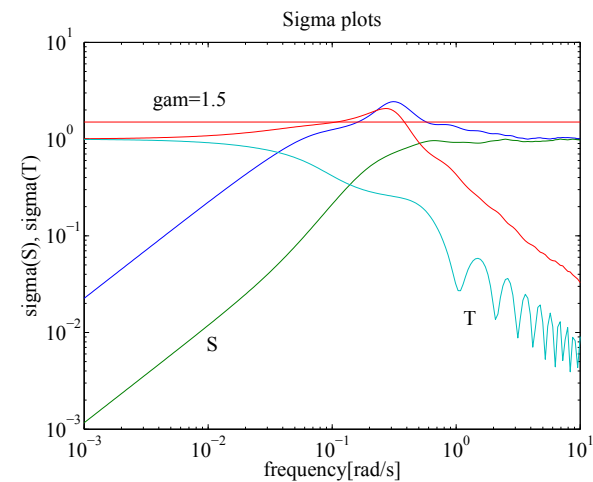

Fig. 10. Singular value plots of $S_{I}$ and $T_{I}$ with PI control
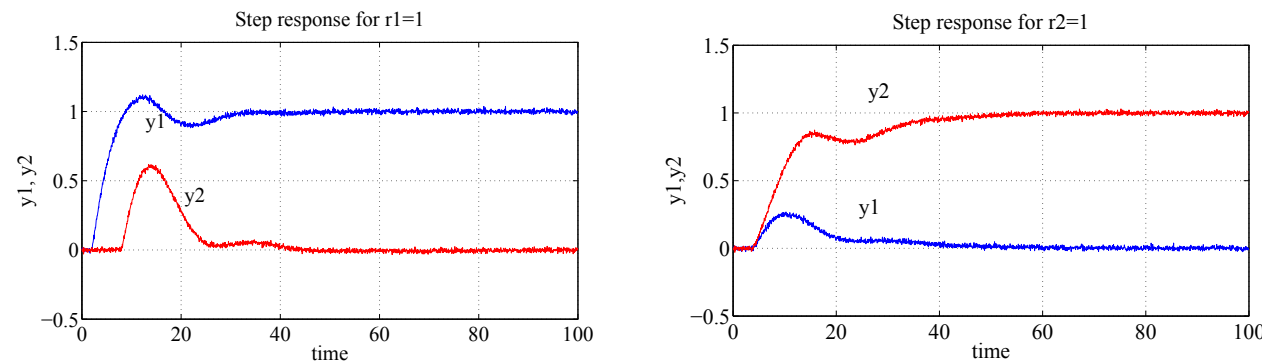

Fig. 11. Output response of the plant for Fig. 12. Output response of the plant for $r_{2}(t)=$ $r_{1}(t)=1$ with PI control 1 with PI control

Next, design a diagonal PID controller with a first order lowpass filter of the next form using the above plant responses. Note that our method can be directly applied to this design problem by considering the plant as $P(s) /(0.1 s+1)$. This filter is used for the attenuation of the loop gain at high frequencies.

$$
K(s)=\frac{1}{0.1 s+1}\left(K_{P}+K_{I} \frac{1}{s}+K_{D^{s}}\right)
$$

Then, we obtain the next controller.

$$
K(s)=\left[\begin{array}{cc}
\frac{0.383 s+0.0798 s+0.477 s^{2}}{(0.1 s+1) s} & 0 \\
0 & \frac{0.118 s+0.0246+0.247 s^{2}}{(0.1 s+1) s}
\end{array}\right] .
$$

Fig. 13 shows the singular value plots, and Fig. 14 and Fig. 15 show the responses of the closed-loop system for the reference inputs. 


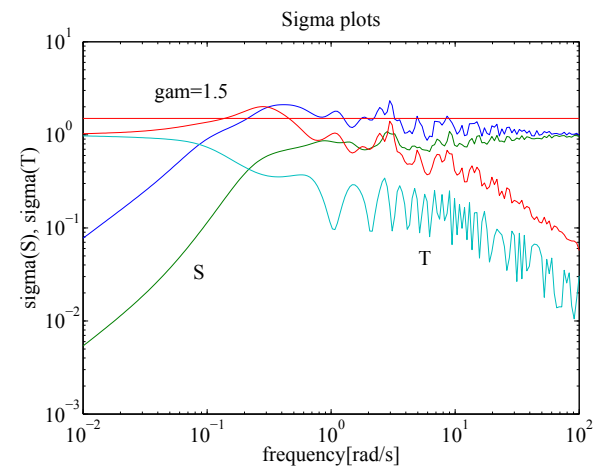

Fig. 13. Singular value plots of $S_{I}$ and $T_{I}$ with PID control

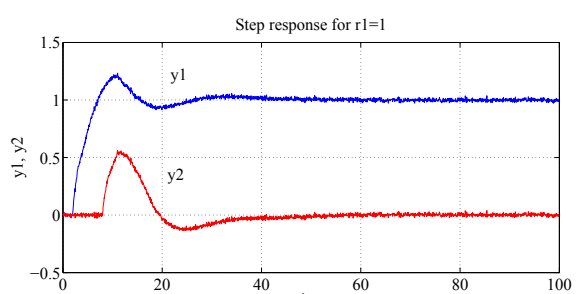

Fig. 14. Output response of the plant for Fig. 15. Output response of the plant $r_{1}(t)=1$ with PID control

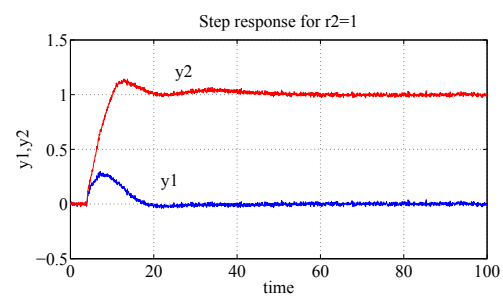

for $r_{2}(t)=1$ with PID control

\section{Experiment using a two-rotor hovering system}

We will design a multi-loop PID controller for a two-rotor hovering system. The general view of our experimental apparatus is shown in Fig.16. The arm $A B$ can rotate around the center $O$ freely, and $y_{1}$ and $y_{2}$ are the yaw and the roll angles, respectively. The airframe $C D$ can also rotate freely on the axis $A B$, and $\theta$ is the pitch angle. Thus, this system has three degrees of freedom. The rotors are driven separately by two DC motors. The rotary encoders are mounted on the joint $O$ to measure the angles $y_{1}$ and $y_{2}[\mathrm{rad}]$, respectively. The encoder for $\theta$ is mounted on the position $A$. The actuator part is illustrated in Fig. 17. The control inputs $u_{1}$ and $u_{2}$ are the thrust and the rolling moment, and $\tilde{f}_{1}$ and $\tilde{f}_{2}$ are the lift forces of the two rotors, respectively. In our previous study, we designed a nonlinear controller for a mathematical model (Saeki \& Sakaue (2001)). Those who are interested in the plant property, please see the reference.

The feedback control system is illustrated in Fig. 18. PID controller $K$ will be designed to track the references $r_{1}, r_{2}$ [rad]. We use a PD controller $0.4+0.2 s /(1+0.01 s)$ in order to control $\theta$, and this gain is determined by trail and error. Then, we treat the plant as a two-input twooutput system. The element denoted by $K_{u v}$ is a constant matrix that transforms the control inputs $u$ to the input voltages $u_{v}$ to the motors. The input voltages are limited to be less than $\pm 5[\mathrm{~V}]$. We consider the subsystem shown by the dotted line as the plant $P$ to be controlled. 


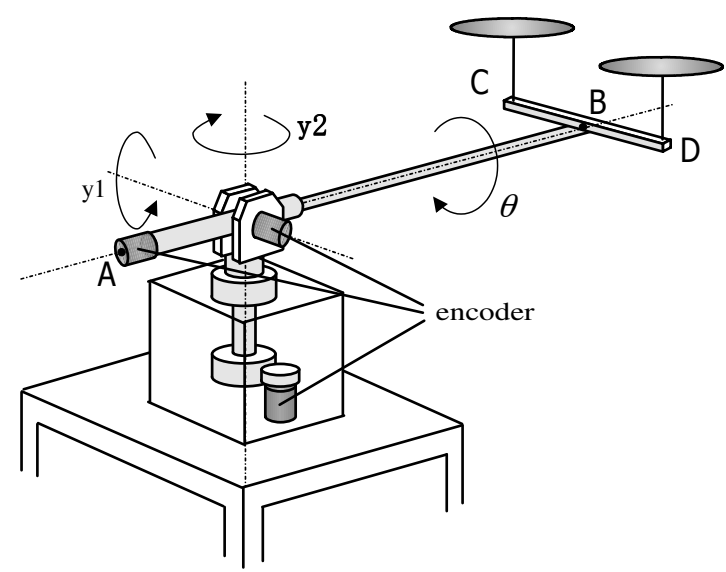

Fig. 16. Experimental setup

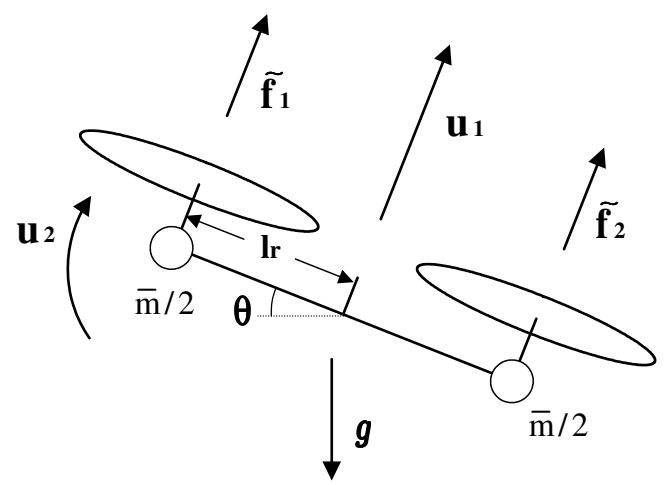

Fig. 17. Illustration of the actuator part

Thus, the feedback system is described by

$$
\begin{aligned}
y & =P(s) e \\
e & =K(s)(r-y)
\end{aligned}
$$

The plant responses shown in Fig. 19 - Fig. 22 are obtained by experiment in the closed-loop operation for the controller

$$
K(s)=\left[\begin{array}{cc}
0.5 & 0 \\
0 & 0.1
\end{array}\right]+\left[\begin{array}{cc}
1 & 0 \\
0 & 0
\end{array}\right] \frac{1}{s}+\left[\begin{array}{cc}
1 & 0 \\
0 & 0.5
\end{array}\right] \frac{s}{0.01 s+1}
$$

Now, let us design a PID controller by using the responses. Since this plant is marginally stable, it is not so easy to give a stabilizing PID controller compared with stable plants. It is 


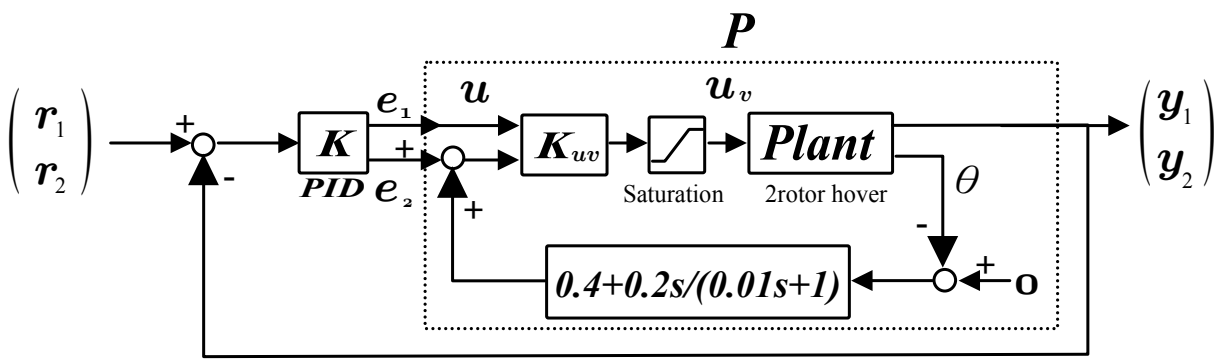

Fig. 18. Feedback control system
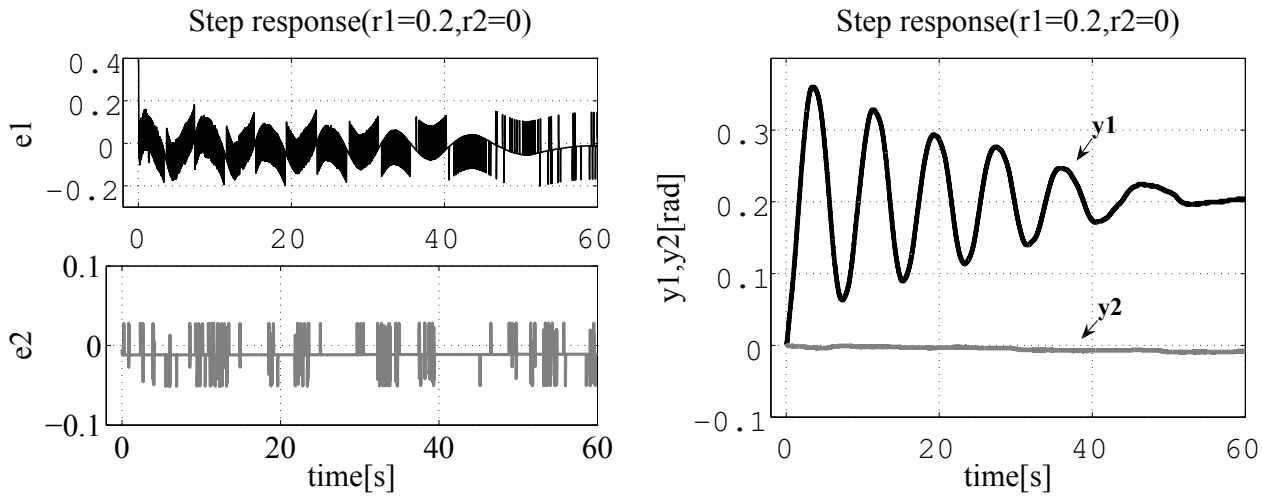

Fig. 19. Input response used for design

Fig. 20. Output response used for design

Step response( $(\mathrm{r} 1=0, \mathrm{r} 2=0.5)$
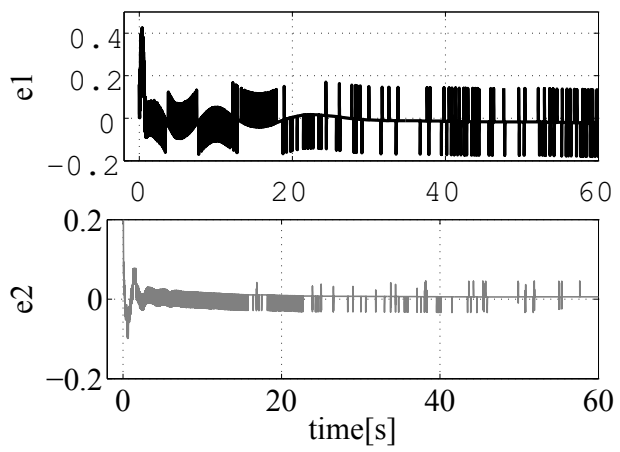

Fig. 21. Input response used for design

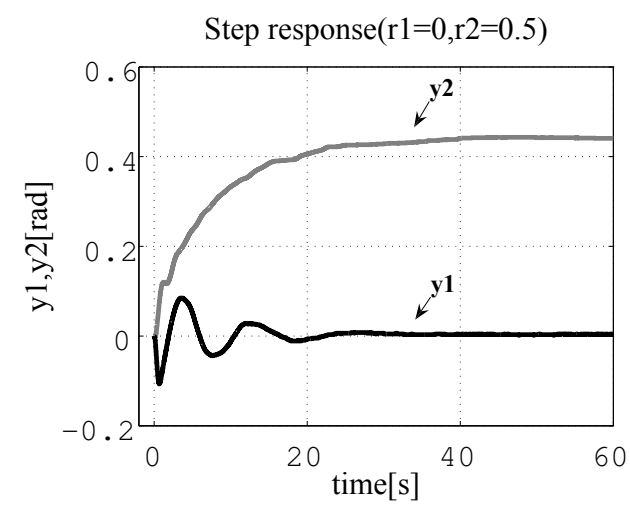

Fig. 22. Output response used for design 
easier to find a stabilizing PD controller than PID controller. Therefore, we give the next PD controller, which is found by trial and error.

$$
K_{a}=\left[\begin{array}{cc}
0.4 & 0 \\
0 & 0.4
\end{array}\right]+\left[\begin{array}{cc}
1 & 0 \\
0 & 0.5
\end{array}\right] s
$$

Sample frequencies $\omega_{i}$ are logarithmically equally spaced 100 points between $10^{-2}$ and $10^{2}$. By solving an LMI once, we obtain the next controller.

$$
K(s)=\left[\begin{array}{cc}
1.4549 & 0 \\
0 & 1.0624
\end{array}\right]+\left[\begin{array}{cc}
0.0980 & 0 \\
0 & 0.1309
\end{array}\right] \frac{1}{s}+\left[\begin{array}{cc}
1.4914 & 0 \\
0 & 1.2581
\end{array}\right] \frac{s}{0.01 s+1}
$$

The step responses are shown in Fig. 23 - Fig. 26. It is necessary to develop an efficient method of finding a stabilizing controller that satisfies (17)(18) for marginally stable or unstable plants. This is our future work.

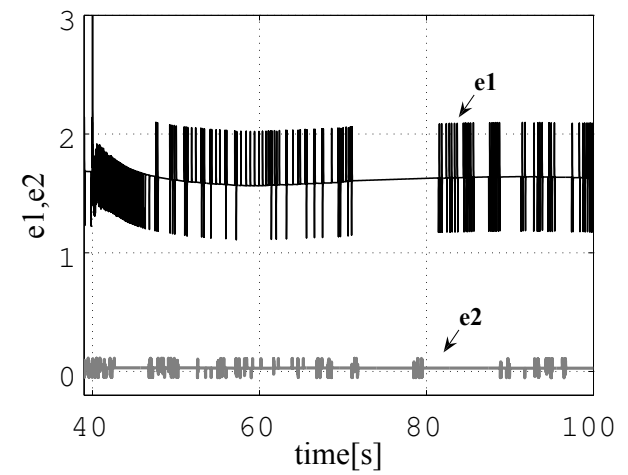

Fig. 23. Input response $(\mathrm{r} 1=0.2, \mathrm{r} 2=0)$

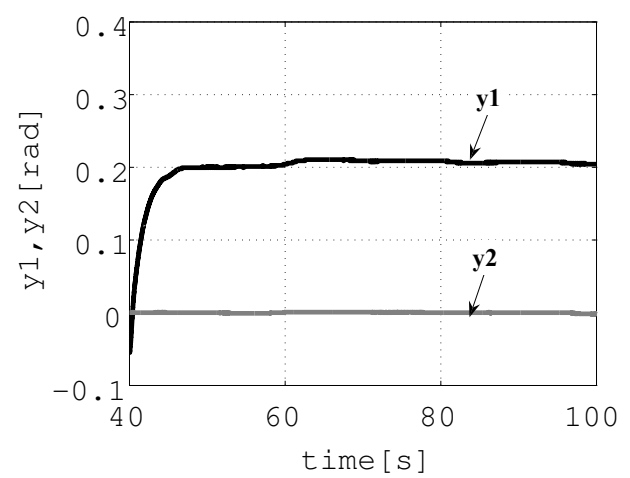

Fig. 25. Output response $1(\mathrm{r} 1=0.2, \mathrm{r} 2=0)$

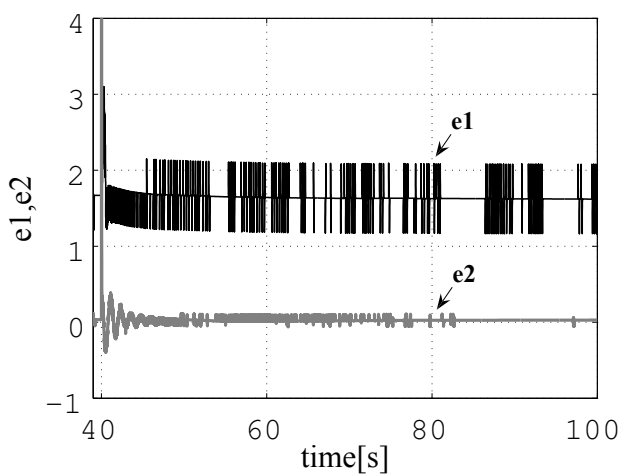

Fig. 24. Input response( $\mathrm{r} 1=0, \mathrm{r} 2=0.5)$

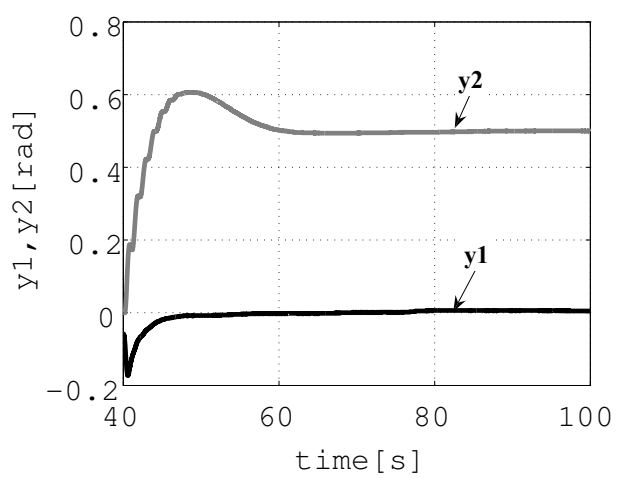

Fig. 26. Output response $2(\mathrm{r} 1=0, \mathrm{r} 2=0.5)$ 


\section{Conclusion}

DDLS (data driven loop shaping method) has been developed for the multi-loop PID control tuning. The constraints on the PID gains are directly derived from a few input-output responses based on falsification conditions without explicitly identifying the plant model. The design problem is reduced to a linear programming or a linear matrix inequality problem, and the solution is obtained by solving it only once.

We have applied our method to the Wood and Berry's binary distillation column process, and our method gives good loop shapes where only two step responses of the closed-loop system are used for design. However, it is difficult to specify the transient response property such as overshoot by our method, because our method treats the optimization problem of disturbance attenuation. Two-degree of freedom control systems may be suitable for the improvement of the transient response. Further, we have applied our method to the control problem of a tworotor hovering system. From our experience including these examples, our method seems considerably robust against noises of the plant input output signals obtained in the closedloop operation. Our design method can be extended to the PID controllers whose gains are full square matrices.

\section{References}

Addison, P.S. (2002). The Illustrated Wavelet Transform Handbook, IOP Publishing Ltd., England. Åström, K \& Hägglund, T (1995). PID Controllers: Theory, Design, and Tuning, ISA, Research Triangle Park, North Carolina.

Åström, K.; Panagopoulous, H.; Hägglund, T.(1998). Design of PI controllers based on nonconvex optimization, Automatica, pp. 585-601.

Åström, K \& Hägglund, T (2006). Advanced PID Control, ISA.

Campi,M.C.; Lecchini, A.; Savaresi, S.M.(2002). Virtual reference feedback tuning: a direct method for the design of feedback controllers, Automatica,Vol. 38, pp. 1337-1346.

Hjalmarsson, H.; Gevers, M.; Gunnarsson, S.;Lequin, O.(1999). Iterative feedback tuning: Theory and application, IEEE Control Systems Magazine, Vol. 42, No. 6, pp. 843-847.

Johnson, M.A. \& Moradi, M.H. (Editors)(2005). PID Control; New identification and design methods, Springer-Verlag London Limited.

Lequin O.; Gevers M.; Mossberg M.; Bosmans E.; Triest L. (2003). Iterative feedback tuning of PID parameters: comparison with classical tuning rules, Control Engineering Practice, Vol. 11, pp. 1023-1033.

Saeki M. \& Sakaue, Y. (2001). Flight control design for a nonlinear non-minimum phase VTOL aircraft via two-step linearization, Proceedings of the 40th IEEE Conf. on Decision and Control, pp. 217-222, Orland, Florida USA.

Saeki, M.(2004a). Unfalsified control approach to parameter space design of PID controllers, Trans. of the Society of Instrument and Control Engineers, Vol. 40, No. 4, pp. 398-404.

Saeki,M.; Hamada, O.; Wada,N.; Masubuchi, I. (2006). PID gain tuning based on falsification using bandpass filters, Proc. of SICE-ICCAS, Busan, Korea, pp. 4032-4037.

Saeki, M.(2008). Model-free PID controller optimization for loop shaping, Proc. of the 17th IFAC World Congress, pp. 4958-4963.

Safonov, M.G. \& Tsao, T.C. (1997). The unfalsified control concept and learning, IEEE Trans. on Automatic Control, Vol. AC-42, No. 6, pp. 843-847.

Skogestad, S. \& Postlethwaite, I. (2007), Multivariable Feedback Control, John Wiley \& Sons, Ltd. 
Vidyasagar, M. (1993). Nonlinear systems analysis, second edition, PRENTICE HALL, Englewood Cliffs.

Wood, R.K. \& Berry, M.W. (1973). Terminal composition control of a binary distillation column, Chem., Eng., Sci., Vo. 28, pp. 1707-1717, 1973. 


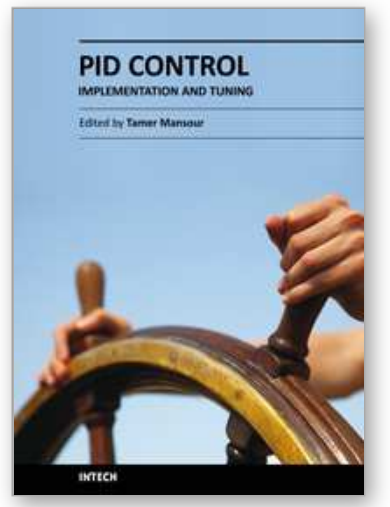

\author{
PID Control, Implementation and Tuning \\ Edited by Dr. Tamer Mansour
}

ISBN 978-953-307-166-4

Hard cover, 238 pages

Publisher InTech

Published online 19, April, 2011

Published in print edition April, 2011

The PID controller is considered the most widely used controller. It has numerous applications varying from industrial to home appliances. This book is an outcome of contributions and inspirations from many researchers in the field of PID control. The book consists of two parts; the first is related to the implementation of PID control in various applications whilst the second part concentrates on the tuning of PID control to get best performance. We hope that this book can be a valuable aid for new research in the field of PID control in addition to stimulating the research in the area of PID control toward better utilization in our life.

\title{
How to reference
}

In order to correctly reference this scholarly work, feel free to copy and paste the following:

Masami Saeki and Ryoyu Kishi (2011). Multi-Loop PID Control Design by Data-Driven Loop-Shaping Method, PID Control, Implementation and Tuning, Dr. Tamer Mansour (Ed.), ISBN: 978-953-307-166-4, InTech, Available from: http://www.intechopen.com/books/pid-control-implementation-and-tuning/multi-loop-pid-controldesign-by-data-driven-loop-shaping-method

\section{INTECH}

open science | open minds

\section{InTech Europe}

University Campus STeP Ri

Slavka Krautzeka 83/A

51000 Rijeka, Croatia

Phone: +385 (51) 770447

Fax: +385 (51) 686166

www.intechopen.com

\section{InTech China}

Unit 405, Office Block, Hotel Equatorial Shanghai

No.65, Yan An Road (West), Shanghai, 200040, China

中国上海市延安西路 65 号上海国际贵都大饭店办公楼 405 单元

Phone: +86-21-62489820

Fax: +86-21-62489821 
(C) 2011 The Author(s). Licensee IntechOpen. This chapter is distributed under the terms of the Creative Commons Attribution-NonCommercialShareAlike-3.0 License, which permits use, distribution and reproduction for non-commercial purposes, provided the original is properly cited and derivative works building on this content are distributed under the same license. 